\title{
Some Definite Integrals of the Product of Two Bessel Functions of the Second Kind: (Order Zero)
}

\author{
By M. L. Glasser
}

\begin{abstract}
A new integral representation is derived for the expression $J_{0}(z) J_{0}(Z)+Y_{0}(z)$ - $Y_{0}(Z)$ and used to evaluate a number of integrals containing $Y_{0}(a x) Y_{0}(b x)$. A supplementary table of integrals involving the function $K_{0}(x)$ in the integrand appears in the microfiche section of this issue.
\end{abstract}

Introduction. There exists a considerable body of information on the subject of integrals of Bessel functions. Of special significance are Chapter XIII of Watson's classic treatise [1] which provides a variety of methods for evaluating such integrals and the excellent book by Luke [2] which provides a thorough summary of results known prior to 1962 . In surveying this material, it came as a considerable surprise that fewer than a dozen integrals containing products of Bessel functions of the second kind are known, not even the simplest one, $\int_{0}^{\infty} Y_{0}(a x) Y_{0}(b x) d x$, nor apparently does there exist any literature dealing with this subject. It therefore appeared to be worthwhile to devote some effort to this problem and the results relating to the case of order zero are described herein. Beside their intrinsic interest, such integrals occur, for example, in the study of wave propagation along a coaxial cable.

Method. We base our considerations on the integral representation

$$
J_{0}(a) J_{0}(b)+Y_{0}(a) Y_{0}(b)=\frac{8}{\pi^{2}} \int_{0}^{\infty} d x \frac{\cos \left[(a-b)\left(x^{2}+1\right)^{1 / 2}\right]}{\left(x^{2}+1\right)^{1 / 2}} K_{0}\left[2 x(a b)^{1 / 2}\right]
$$

which is valid for positive real $a$ and $b$. Equation (1) can be derived in a variety of ways; the derivation presented here has been chosen because it is short and novel. It can be shown that the Dirac delta distribution has the integral representation [3]

$$
\delta(x)=\pi^{-2} x \sinh (\pi x) \int_{0}^{\infty} d t t^{-1} K_{0}(t) K_{i x}(t)
$$

Hence, by the Parseval theorem for the Kontorovich-Lebedev transform [4], when both sides exist

$$
\int_{0}^{\infty} d t t^{-1} K_{0}(t) f(x)=\frac{\pi}{4} g^{\prime \prime}(0)
$$

where $g$ is the inverse K-L image of $f$. From Eqs. (10)-(16) of reference [5], we obtain

Received May 3, 1973.

AMS (MOS) subject classifications (1970). Primary 33A40; Secondary 44A20.

Key words and phrases. Bessel function, definite integrals.

Copyright @ 1974, American Mathematical Society 


$$
\begin{aligned}
\int_{0}^{\infty} d x x \sinh (\pi x)\left\{J_{i x}(a) J_{i x}(b)+Y_{i x}(a) Y_{i x}(b)\right\} K_{2 i x}(y) \\
=y\left(y^{2}+4 a b\right)^{-1 / 2} \cos \left\{\frac{1}{2}\left[(a-b) /(a b)^{1 / 2}\right]\left(y^{2}+4 a b\right)^{1 / 2}\right\} .
\end{aligned}
$$

Expressing (4) as a K-L transform and applying (3), Eq. (1) results directly, after an elementary substitution.

Examples. (A) As an elementary consequence of (1), we find, with $t^{2}=4 a b$, $a-b=2 \alpha(a b)^{1 / 2}, a>b$,

$$
\begin{aligned}
\int_{0}^{\infty} d \alpha F(\alpha)\left\{J_{0}\left(\frac{1}{2} t c\right) J_{0}\left(\frac{1}{2} t d\right)+Y_{0}\left(\frac{1}{2} t c\right) Y_{0}\left(\frac{1}{2} t d\right)\right\} \\
=\frac{8}{\pi} \int_{t}^{\infty} d x F_{c}(x) K_{0}\left[\left(x^{2}-t^{2}\right)^{1 / 2}\right] /\left(x^{2}-t^{2}\right)^{1 / 2}
\end{aligned}
$$

with $c=\left(\alpha^{2}+1\right)^{1 / 2}+\alpha, d=\left(\alpha^{2}+1\right)^{1 / 2}-\alpha$ and where $F_{c}$ denotes the Fourier cosine transform of $F$. Thus, we find, on taking $F(\alpha)=\cos (\alpha u), F(\alpha)=\left(\alpha^{2}+u^{2}\right)^{-1}$, respectively,

$$
\begin{aligned}
& \int_{t}^{\infty} d x \cos (x \alpha) \frac{K_{0}\left[\left(x^{2}-t^{2}\right)^{1 / 2}\right]}{\left(x^{2}-t^{2}\right)^{1 / 2}}=\frac{\pi^{2}}{8}\left\{J_{0}\left(\frac{1}{2} t c\right) J_{0}\left(\frac{1}{2} t d\right)+Y_{0}\left(\frac{1}{2} t c\right) Y_{0}\left(\frac{1}{2} t d\right)\right\} \\
& \int_{0}^{\infty} \frac{d x}{x^{2}+\alpha^{2}}\left\{J_{0}\left[t\left[\left(x^{2}+1\right)^{1 / 2}+x\right]\right] J_{0}\left[t\left[\left(x^{2}+1\right)^{1 / 2}-x\right]\right]\right. \\
& \left.+Y_{0}\left[t\left[\left(x^{2}+1\right)^{1 / 2}+x\right]\right] Y_{0}\left[t\left[\left(x^{2}+1\right)^{1 / 2}-x\right]\right]\right\} \\
& =\frac{2}{\pi \alpha} K_{0}\left\{t\left[\alpha+\left(\alpha^{2}-1\right)^{1 / 2}\right]\right\} K_{0}\left\{t\left[\alpha-\left(\alpha^{2}-1\right)^{1 / 2}\right]\right\}, \quad \operatorname{Re} \alpha>1 .
\end{aligned}
$$

(B) In (1), let $a \rightarrow a t, b \rightarrow b t$. Then, multiplying both sides by $\cos (t y)$, noting that integrating over all positive $t$ can precede the $x$-integrations on the right-hand side and using the formulas

$$
\begin{aligned}
& \int_{0}^{\infty} d x K_{0}(x) Y_{0}(a x)=-\left(a^{2}+1\right)^{-1 / 2} K\left[\left(a^{2}+1\right)^{-1 / 2}\right], \\
& \int_{0}^{\infty} d x K_{0}(x) K_{0}(a x)=\pi(a+1)^{-1} K(|a-1| /(a+1)),
\end{aligned}
$$

we obtain the Fourier cosine pair

$$
\begin{aligned}
& \int_{0}^{\infty} d t \cos (t u)\left\{J_{0}(a t) J_{0}(b t)+Y_{0}(a t) Y_{0}(b t)\right\}(4 / \pi)\left[(a+b)^{2}-u^{2}\right]^{-1 / 2} K\left\{\left[4 a b /\left[(a+b)^{2}-u^{2}\right]\right]^{1 / 2}\right\}, \quad 0<u<|a-b|, \\
&=(8 / \pi)\left\{\left[u^{2}-(a-b)^{2}\right]^{1 / 2}+2(a b)^{1 / 2}\right\}^{-1} K\left\{\left|\frac{\left[u^{2}-(a-b)^{2}\right]^{1 / 2}-2(a b)^{1 / 2}}{\left[u^{2}-(a-b)^{2}\right]^{1 / 2}+2(a b)^{1 / 2}}\right|\right\} \\
& u>|a-b|
\end{aligned}
$$

By combining this with Bailey's results for the first integral on the left-hand side of (9), we obtain $\int_{0}^{\infty} d t \cos (t u) Y_{0}(a t) Y_{0}(b t)$ for a number of cases. Thus, in general, 
taking $u=0$ in (9),

$$
\frac{\pi}{2} \int_{0}^{\infty} Y_{0}(a x) Y_{0}(b x) d x=\frac{2}{a+b} K\left(\frac{2(a b)^{1 / 2}}{a+b}\right)-a^{-1} K(b / a), \quad a>b .
$$

In conclusion, we present two other results which can be obtained in the same manner:

$$
\begin{aligned}
& \int_{0}^{\infty} d t t^{\nu+1} J_{\nu}(t u)\left\{J_{0}(a t) J_{0}(b t)+Y_{0}(a t) Y_{0}(b t)\right\} \\
& =4 \pi^{-3 / 2} \frac{\Gamma^{2}(\nu+1)}{\Gamma(\nu+3 / 2)} \frac{u^{\nu}}{\left[u^{2}-(a-b)^{2}\right]^{p+1}} \\
& \quad{ }_{2} F_{1}\left(\nu+1, \frac{1}{2} ; \nu+3 / 2 ; \frac{u^{2}-(a+b)^{2}}{u^{2}-(a-b)^{2}}\right), \quad u>(a-b) . \\
& \frac{\pi^{2}}{8} \int_{0}^{\infty} d t t J_{0}(t u)\left\{J_{0}(a t) J_{0}(b t)+Y_{0}(a t) Y_{0}(b t)\right\} \\
& =\frac{\ln \left\{2(a b)^{1 / 2} /\left[\left[(a-b)^{2}-u^{2}\right]^{1 / 2}+\left[(a+b)^{2}-u^{2}\right]^{1 / 2}\right]\right\}}{\left[(a-b)^{2}-u^{2}\right]^{1 / 2}\left[(a+b)^{2}-u^{2}\right]^{1 / 2}}, \quad u<|a-b|, \\
& =\frac{\sin ^{-1}\left[\left[(a+b)^{2}-u^{2}\right]^{1 / 2} / 2(a b)^{1 / 2}\right]}{\left[u^{2}-(a-b)^{2}\right]^{1 / 2}\left[(a+b)^{2}-u^{2}\right]^{1 / 2}}, \quad|a-b|<u<(a+b), \\
& =\frac{\sinh ^{-1}\left[\left[u^{2}-(a+b)^{2}\right]^{1 / 2} / 2(a b)^{1 / 2}\right]}{\left[u^{2}-(a-b)^{2}\right]^{1 / 2}\left[u^{2}-(a+b)^{2}\right]^{1 / 2}}, \quad(a+b)<u .
\end{aligned}
$$

Equation (12) combined with Bailey's formula

$$
\begin{aligned}
\int_{0}^{\infty} t J_{0}(t u) J_{0}(a t) J_{0}(b t) d t & =(2 \pi \Delta)^{-1}, & & a, b, u \text { form a triangle of area } \Delta, \\
& =0, & & \text { otherwise, }
\end{aligned}
$$

gives $\int_{0}^{\infty} d t t J_{0}(u t) Y_{0}(a t) Y_{0}(b t)$.

These results can probably be extended to $Y_{\nu}(a x) Y_{\nu}(b x), \nu \neq 0$, by an appropriate generalization of (4), but as yet we have no approach to the case $Y_{\nu}(a x) Y_{\mu}(b x)$ except when $\mu-\nu$ is an integer and the recursion relations can be applied.

A number of other interesting integrals which can be derived in a manner similar to (1) have been included in a collection of new integrals of the function $K_{0}$ which is reproduced in the microfiche section of this issue.

Battelle Memorial Institute

Columbus, Ohio 43201

1. G. N. Watson, A Treatise on the Theory of Bessel Functions, 2nd ed., Cambridge Univ. Press, Cambridge; Macmillan, New York, 1944. MR 6, 64. \#5198.

2. Y. L. LuKE, Integrals of Bessel Functions, McGraw-Hill, New York, 1962. MR 25

3. See, e.g., G. Z. Forristall \& J. D. Ingram, SIAM J. Math. Anal., v. 3, 1972, p. 561.

4. A. ERDÉLYI ET AL., Tables of Integral Transforms. Vol. 2, McGraw-Hill, New York, 1954, p. 173. MR 16, 468.

5. Ibid, p. 380 . 